\title{
ON KY FAN'S MINIMAX PRINCIPLE
}

\author{
E. TARAFDAR and H. B. THOMPSON
}

(Received 23 January; revised 9 April 1977)

Communicated by N. Smythe

\begin{abstract}
A generalized version of the Knaster-Kuratowski-Mazurkiewicz theorem is obtained and used to generalize Ky Fan's minimax principle. This result is applied to a variational inequality.

Subject classification (Amer. Math. Soc. (MOS) 1970): primary 47H05, secondary 47H10.
\end{abstract}

\section{Introduction}

In Ky Fan (1972) Ky Fan has proved a minimax principle by using his own generalized version (Ky Fan (1961)) of Knaster - Kuratowski - Mazurkiewicz's theorem. In a joint paper Brezis, Nirenberg, and Stampacchia (1972) have given a further extension of Knaster-Kuratowski-Mazurkiewicz's theorem and applied this extended theorem to a number of problems including a generalized Ky Fan's minimax principle. In this note we have obtained a result which is analogous to the extended Knaster-KuratowskiMazurkiewicz theorem of Brezis-Nirenberg-Stampacchia. Using our result we have proved a Ky Fan's minimax principle which includes the corresponding theorem of Brezis-Nirenberg-Stampacchia. We have also shown that our result is also applicable to the types of problems considered in Brezis, Nirenberg, and Stampacchia (1972). Our approach is via a simple fixed point theorem of Browder (1968) and is different from that in Brezis, Nirenberg, and Stampacchia (1972) and Ky Fan (1972).

The authors wish to thank Professor R. Vyborny for suggesting the topic.

In the sequel, $E$ will denote a Hausdorff topological vector space. For any finite subset $\left\{x_{1}, x_{2}, \cdots, x_{n}\right\}$ of $E,\left\langle x_{1}, x_{2}, \cdots, x_{n}\right\rangle$ will denote the convex 
hull of $\left\{x_{1}, x_{2}, \cdots, x_{n}\right\}$. We first consider the following lemma (see Brezis, Nirenberg, and Stampacchia (1972), p. 2).

Lemma 2.1. Let $X$ be a nonempty subset of $E$. To each $x \in X$, let a nonempty subset $F(x)$ of $E$ be given such that

(i) $\overline{F\left(x_{0}\right)}=L$ is compact for some $x_{0} \in X$;

(ii) the convex hull of every finite subset $\left\{x_{1}, x_{2}, \cdots, x_{n}\right\}$ of $X$ is contained in the corresponding union $\bigcup_{i=1}^{n} F\left(x_{i}\right)$;

(iii) for each $x \in X$, the intersection of $F(x)$ with any finite dimensional subspace is closed;

(iv) for every convex subset $D$ of $E$ the following equality holds

$$
\left\{\widehat{\bigcap}_{x \in X \cap D} F(x)\right) \cap D=\left(\bigcap_{x \in X \cap D} F(x)\right) \cap D .
$$

Then $\bigcap_{x \in X} F(x) \neq \phi$.

The above lemma is a slight generalization of Ky Fan's generalization (see Ky Fan (1961) Lemma 1, p. 305) of the well known classical finite dimensional result of Knaster-Kuratowski-Mazurkiewicz (1929).

To obtain our lemma we shall use the following fixed point theorem of Browder (1968), Theorem 1, p. 285.

THEOREM 2.1. (Browder). Let $K$ be a compact convex subset of E. Let $T$ be a multi-valued mapping of $K$ into $2^{K}$ such that

(i) for each $x \in K, T(x)$ is a nonempty convex subset of $K$;

(ii) for each $x \in K, T^{-1}(x)=\{y \in K: x \in T(y)\}$ is open in $K$.

Then there is a point $x_{0} \in K$ such that $x_{0} \in T\left(x_{0}\right)$.

We now prove the following preliminary lemma.

Lemma 2.2. Let $X$ be a nonempty subset of $E$. To each $x \in X$, let a nonempty set $F(x)$ of $E$ be given such that

(a) $x \in F(x)$ for each $x \in F(x)$;

(b) $F\left(x_{0}\right)$ is compact for some $x_{0} \in X$;

(c) for each finite subset $\left\{x_{1}, x_{2}, \cdots, x_{n}\right\}$ of $X$ and each $x \in S_{n}=$ $\left\langle x_{1}, x_{2}, \cdots, x_{n}\right\rangle=$ the convex hull of $\left\{x_{1}, x_{2}, \cdots, x_{n}\right\}$, the set $A(x)=$ $\left\{y \in S_{n} \cap X: x \notin F(y)\right\}$ has the property that whenever $A(x)$ is nonempty, it contains a nonempty convex subset $H(x)$ such that the set $P(x)=$ $\left\{y \in S_{n}: x \notin H(y)\right\}$ is closed;

(d) $F\left(x_{0}\right) \cap F(x)$ is closed for each $x \in C$.

Then $\bigcap_{x \in X} F(x) \neq \phi$.

Proof. In view of (b) and (d) it suffices to prove that $\bigcap_{i=1}^{n} F\left(x_{i}\right) \neq \phi$ for each finite subset $\left\{x_{1}, x_{2}, \cdots, x_{n}\right\}$ of $X$. On the contrary we suppose that for 
some finite subset $\left\{x_{1}, x_{2}, \cdots, x_{k}\right\}$ of $X$ we have $\bigcap_{i=1}^{k} F\left(x_{i}\right)=\phi$. Then for each $x \in S_{k}=\left\langle x_{1}, x_{2}, \cdots, x_{k}\right\rangle$ the set $A(x)=\left\{y \in S_{k} \cap X: x \notin F(y)\right\}$ is nonempty. Indeed, at least one of the points $x_{i}, i=1,2, \cdots, k$ must be in $A(x)$, for otherwise $\bigcap_{i=1}^{k} F\left(x_{i}\right)$ would be nonempty. We now define a multi-valued mapping $T: S_{k} \rightarrow 2^{S_{k}}$ by $T(x)=H(x), x \in S_{k} ; T$ is well defined by virtue of (c). Now for each $x \in S_{k}, T^{-1}(x)=\left\{y \in S_{k}: x \in T(y)\right\}=\left\{y \in S_{k}: x \in H(y)\right\}=$ complement of $P(x)$ in $S_{K}$ which is an open set in $S_{k}$ by condition (c) $(P(x)$ being closed in $S_{k}$ ). Hence by the fixed point theorem of Bruwder there is a point $x_{0} \in S_{k}$ such that $x_{0} \in T\left(x_{0}\right)$. But then by definition of $T\left(x_{0}\right)$ we have $x_{0} \notin F\left(x_{0}\right)$ which contradicts (a). Thus $\bigcap_{i=1}^{k} F\left(x_{i}\right) \neq \phi$.

We are now in a position to prove our main lemma.

Lemma 2.3. Let $X$ be a nonempty subset of $E$. To each $x \in X$, let $a$ nonempty subset $F(x)$ of $E$ be given such that

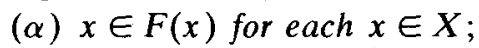

(B) $\overline{F\left(x_{0}\right)}=L$ is compact for some $x_{0} \in X$;

$(\gamma)$ for each finite subset $\left\{x_{1}, x_{2}, \cdots, x_{n}\right\}$ of $X$ and each $x \in S_{n}=$ $\left\langle x_{1}, x_{2}, \cdots, x_{n}\right\rangle$ the set $A(x)=\left\{y \in S_{n} \cap X: x \notin F(y)\right\}$ has the same property as laid down in (c) of Lemma 2.2 .

( $\delta)$ for each $x \in X$, the intersection of $F(x)$ with any finitc dimensional subspace is closed;

( $\omega)$ the Brezis-Nirenberg-Stampacchia condition holds, that is, for every convex subset $D$ of $E$ we have $\left(\widehat{\bigcap}_{x \in X \cap D} F(x)\right) \cap D=\left(\bigcap_{x \in X_{1} D} F(x)\right) \cap D$.

Then $\bigcap_{x \in X} F(x) \neq \phi$.

Proof. We may assume $x_{0}=0$. Let $\left(E_{i}\right)_{i \in I}$ be the class of all finite dimensional subspaces of $E$ ordercd by inclusion i.e. $i \geqq j$ means $E_{j} \subset E_{i}$. Restricting to $E_{i}$ the conditions of Lemma 2.2 apply to $X_{i}=X \cap E_{i}$ and $F_{i}(x)=F(x) \cap E_{i}$. Clearly (a) and (c) are satisfied and (b) and (d) follow from $(\beta)$ and $(\delta)$. By Lemma 2.2 there is $u_{i} \in L \cap E_{i}$ satisfying

$$
u_{i} \in F_{i}(x) \subset F(x) \text { for every } x \in X_{i} .
$$

We now repeat the argument of Brezis, Nirenberg, and Stampacchia (1972). Let $\phi_{i}=\bigcup_{j \geq i}\left\{u_{j}\right\}$ and so $u \in F(z)$ for $u \in \phi_{i}$ and $z \in x_{i}$ and hence $\phi_{i} \subset$ $\bigcap_{z \in x_{i}} F(z)$.

Suppose $\tilde{x} \in \bigcap_{i \in I} \bar{\phi}_{i}$-which is non-empty since $\bar{\phi}_{i} \subset L$ is compact and let $i_{0}$ be such that $\tilde{x} \in E_{i_{0}}$. For any $x \in X$ we can find $i \geqq i_{0}$ such that $x \in E_{i}$. We have

$$
\tilde{x} \in \bar{\phi}_{i} \cap E_{i} \subset\left(\overline{\bigcap_{z \in X_{i}} F(z)}\right) \cap E_{i}=\bigcap_{z \in X_{i}} F_{i}(z)
$$

by $(\omega)$. Therefore $\tilde{x} \in F_{i}(x) \subset F(x)$ and consequently $\tilde{x} \in \bigcap_{x \in X} F(x)$. 


\section{Comparison between Lemma 2.1 and Lemma 2.3}

(A). If condition $(\gamma)$ of Lemma 2.3 is strengthened to the condition: $(\gamma)^{\prime}$ for each $x \in S_{n}=\left\langle x_{1}, x_{2}, \cdots, x_{n}\right\rangle$ the set $A(x)=$ $\left\{y \in S_{n} \cap X: x \notin F(y)\right\}$ is convex, then Lemma 2.3 follows from Lemma 2.1.

To show this, it is enough to show that $(\gamma)^{\prime}$ implies condition (ii) of Lemma 2.1. Let $(\alpha)$ hold and $\left\{x_{1}, x_{2}, \cdots, x_{n}\right\}$ be any finite subset of $X$. Suppose (ii) fails and $S_{n}=\left\langle x_{1}, x_{2}, \cdots, x_{n}\right\rangle \notin \bigcup_{i=1}^{n} F\left(x_{i}\right)$. Then there is $x \in S_{n}$ with $x \notin \bigcup_{i=1}^{n} F\left(x_{i}\right), \quad x=\sum_{i=1}^{n} \lambda_{i} x_{i}, \lambda_{i} \geqq 0$, and $\sum_{i=1}^{n} \lambda_{i}=1$. Since $x \notin F\left(x_{i}\right), x_{i} \in$ $A(x)$ for all $i=1,2, \cdots, n$, and hence $x=\sum_{i=1}^{n} \lambda_{i} x_{i} \in A(x)$ by $(\gamma)^{\prime}$. This means that $x \notin F(x)$ contradicting $(\alpha)$. Thus (ii) of Lemma 2.1 and Lemma 2.3 follows from Lemma 2.1 .

Remark. It is interesting to note that in this case we can take $H(x)=A(x)$ for each $x \in X$ since $P(x)=\left\{y \in S_{n}: x \notin H(y)=A(y)\right\}=$ $\left\{y \in S_{n}: x \notin F(y)\right\}$ is automatically closed by $(\delta)$.

(B). Lemma 2.1 applies to the following example although Lemma 2.3 does not apply.

Let $E$ be the plane $R^{2}, S=\left\{(u, v) \in R^{2}:-1 \leqq u, v \leqq 1\right\}$, and $X=$ $\{(u, v) \in S:|u|=|v|=1\}$. For $x=(i, j) \in X$ set $F(x)=\left\{(u, v) \in R^{2}: 0 \leqq\right.$ $i u, j v \leqq 1\}$. Clearly Lemma 2.1 applies and by inspection $\left.\bigcap_{x \in X} F(x)=\{0,0)\right\}$. That Lemma 2.3 does not apply can be seen as follows. For $x$ in $S$ let $A(x)=\{y \in S \cap X: x \notin F(y)\}$ so that for $x \neq(0,0), A(x)$ is a non-empty subset of $X$. Let $H(x)$ be a non-empty convex subset of $A(x)$ for $x \neq(0,0)$. Suppose $H^{-1}(x)=\{y \in S: x \in H(y)\}$ is open in $S$ for all $x$ in $S$. Now $H^{-1}(x)$ is empty for $x$ not in $X$ and since $H(x)$ is a single element for $x \neq(0,0)$ non-empty $H^{-1}(x)$ are disjoint. Now $\bigcup_{x \in X} H^{-1}(x)=S-\{(0,0)\}$ is connected which is a contradiction.

(C). Lemma 2.3 applies to the following example although Lemma 2.1 does not apply.

Let $E$ be the reals, $F(-3)=\{x \in R:-3 \leqq x \leqq-2$ or $|x| \leqq 1\}$ and $F(3)=\{x \in R: 2 \leqq x \leqq 3$ or $|x| \leqq 1\}$. Clearly Lemma 2.1 does not apply since $[-3,3]$ is not a subset of $F(3) \cup F(-3)$. Now Lemma 2.3 applies since for $x$ in $[-3,3], A(x)=\{y \in[-3,3] \cap\{-3,3\}: x \notin F(y)\}$ and we may choose

$$
H(x)=\left\{\begin{aligned}
-3, & \text { for } x>1 \\
3, & \text { for } x<-1
\end{aligned}\right.
$$

Then $H(x)$ is a convex subset of $A(x)$ and $H^{-1}(x)$ is open in $[-3,3]$. The other conditions of Lemma 2.3 are clearly satisfied. 


\section{Applications}

THEOREM 4.1. (Minimax priciple). Let $K$ be a non-empty convex subset of $E$ and $f(x, y)$ be a real valued function defined on $K \times K$ such that

(i) $f(x, x) \leqq 0$ for each $x \in K$;

(ii) for each finite subset $\left\{x_{1}, x_{2}, \cdots, x_{n}\right\}$ of $K$ and $x \in S_{n}=\left\langle x_{1}, x_{2}, \cdots, x_{n}\right\rangle$ the set $A(x)=\left\{y \in S_{n}: f(x, y)>0\right\}$ if non-empty contains a non-empty convex subset $H(x)$ such that the set

$$
P(x)=\left\{y \in S_{n}: x \notin H(y)\right\}
$$

is closed;

(iii) for each $y \in K, f(x, y)$ is a lower semicontinuous function of $x$ on the intersection of $K$ with any finite dimensional subspace of $E$;

(iv) there is a compact subset $L$ of $E$ and $y_{0} \in L \cap K$ such that $f\left(x, y_{0}\right)>0$ for $x \in K, x \notin L$;

(v) whenever $x, y \in K$ and $x_{\alpha}$ is a net on $K$ converging to $x$, then $f\left(x_{\alpha},(1-t) x+t y\right) \leqq 0$ for every $t \in[0,1]$ implies $f(x, y) \leqq 0$.

Then there is a point $x_{0} \in L \cap K$ such that

$$
f\left(x_{0}, y\right) \leqq 0 \text { for all } y \in K .
$$

In particular, $\operatorname{Inf}_{x \in K} \sup _{y \in K} f(x, y) \leqq 0$.

Proof. For each $z \in K$ we set

$$
F(z)=\{x \in K: f(x, z) \leqq 0\} .
$$

For each finite subset $\left\{x_{1}, x_{2}, \cdots, x_{n}\right\}$ of $K$ and $x \in S_{n}=\left\langle x_{1}, x_{2}, \cdots, x_{n}\right\rangle$ the set $A(x)=\left\{y \in S_{n}: x \notin F(y)\right\}=\left\{y \in S_{n}: f(x, y)>0\right\}$ has the property $(\gamma)$ of Lemma 2.3 by (ii). While $(\alpha),(\delta)$ and $(\omega)$ of Lemma 2.3 follow from (i), (iii) and $(v)$ respectively (to see that $(v)$ implies $(\omega)$ we refer to proof of application 2, Brezis, Nirenberg, and Stampacchia (1972), p. 4. Finally by (iv), $F\left(y_{0}\right)$ is compact and hence we have $(\beta)$ of Lemma 2.3. Thus by Lemma 2.3 there is a point $x_{0} \in L \cap K$ such that

$$
x_{0} \in \bigcap_{x \in K} F(x), \text { that is, } f\left(x_{0}, y\right) \leqq 0 \text { for all } y \in K
$$

We note that $x_{0} \in L$ by virtue of (iv).

Corollary 4.1. (Brezis Nirenberg and Stampacchia (1972)). Let K be $a$ non-empty convex subset of $E$ and $f(x, y)$ be a real valued function defined on $\mathrm{K} \times \mathrm{K}$ such that

(i) $f(x, x) \leqq 0$ for each $x \in K$;

(ii)' for every $x \in K$, the set $\{y \in K: f(x, y)>0\}$ is convex; 
(iii)' the condition (iii) of Theorem 3.1 holds;

(iv)' the condition (iv) of Theorem 3.1 holds;

(v)' the condition (v) of Theorem 3.1 holds.

Then there exists a point $x_{0} \in L \cap K$ such that

$$
f\left(x_{0}, y\right) \leqq 0 \text { for all } y \in K .
$$

Proof. As before we set

$$
F(z)=\{x \in K: f(x, z) \leqq 0\} \text { for each } z \in K .
$$

The set $A^{\prime}(x)=\{y \in K: f(x, y)>0\}$ is convex by (ii)'. Hence for any finite subset $\left\{x_{1}, x_{2}, \cdots, x_{n}\right\}$ of $K$ and $x \in S_{n}=\left\langle x_{1}, x_{2}, \cdots, x_{n}\right\rangle$ the set $A(x)=$ $\left\{y \in S_{n}: f(x, y)>0\right\}$ is convex. Now we choose $H(x)=A(x)$ for each $x \in K$. The set $P(x)=\left\{y \in S_{n}: x \in H(y)\right\}$ is closed by (iii)' because of the reason given in the remark following (A). Thus the conclusion of the corollary follows from the Theorem 4.1.

Corollary 4.2. (Ky Fan (1972)). Let $K$ be a non-empty compact convex subset of $E$ and $f(x, y)$ be a real valued function defined on $K \times K$ such that

(0) $f(x, x) \leqq 0$ for each $x \in K$;

(00) for each $x \in K$, the set $\{y: f(x, y)>0\}$ is convex;

$(000)$ for each $y \in K, f(x, y)$ is a lower semicontinuous function of $x$ on $K$. Then there is a point $x_{0} \in K$ such that $f\left(x_{0}, y\right) \leqq 0$ for all $y \in K$.

Proor. This follows from Corollary 4.1.

Let $E$ be Hausdorff topological vectors space over the reals and $K$ be a subset of $E$. Then a mapping $A$ of $K$ into $E^{*}$ is called pseudomonotone if, whenever $x_{\alpha}$ is a net in $K$ converging to $x$ with $\lim \sup \left(A x_{\alpha}, x_{\alpha}-x\right) \leqq 0$ then $\lim \inf \left(A x_{\alpha}, x_{\alpha}-y\right) \geqq(A x, x-y)$. Here $(.,$.$) denotes the pairing between E^{*}$ and $E$.

Corollary 4.3. (Brezis (1968), Corollary 29). Let $K$ be convex subset of $E$ (over reals) and let $f(x, y)=(A x, x-y)+\phi(x)-\phi(y)$ where $A$ is a pseudo-monotone mapping from $K$ into $E^{*}$ and $\phi$ is a lower semicontinuous convex function. In addition we assume that $A$ is continuous from any finite dimensional subspace of $E$ to the weak topology of $E^{*}$ and condition (iv)' of Corollary 4.1 holds. Then there exists $x_{0} \in L \cap K$ such that $\left(A x_{0}, x_{0}-y\right)+$ $\phi\left(x_{0}\right)-\phi(y) \leqq 0$ for all $y \in K$.

Proof. The conditions (i)', (ii)', and (iii)' of Corollary 4.1 follow immediately. To verify that (v)' holds, see the proof of application 3, Brezis, Nirenberg, and Stampacchia (1972), p. 5. 


\section{References}

H. Brezis (1968), 'Equations et inequations nonlineaires dans les espaces vectoriels en dualite', Ann. Inst. Fourier (Grenoble) 18, 115-175.

H. Brezis, L. Nirenberg, and G. Stampacchia (1972), 'A remark on Ky Fan's Minimax principle', Bollentino U.M. Italiana (4) 6, 293-300.

F. Browder (1968), 'The fixed point theory of multi-valued mappings in topological vector spaces', Math. Annalen 177, 177-283.

K. Fan (1961), 'A generalization of Tychonoff's fixed point theorem', Math. Ann. 142, 305-310.

K. Fan (1972), 'A minimax inequality and applications', Inequalities III, edited O. Shisha (Academic Press).

B. Knaster, C. Kuratowski, and S. Mazurkiewicz (1929), 'Ein Beweis des Fixpunktsatzes fur n-dimensionale Simplexe', Fund. Math. 14, 132-137.

Department of Mathematics,

University of Queensland,

St. Lucia. Queensland 4067, Australia. 